\title{
Article \\ Podoplanin drives dedifferentiation and amoeboid invasion of melanoma
}

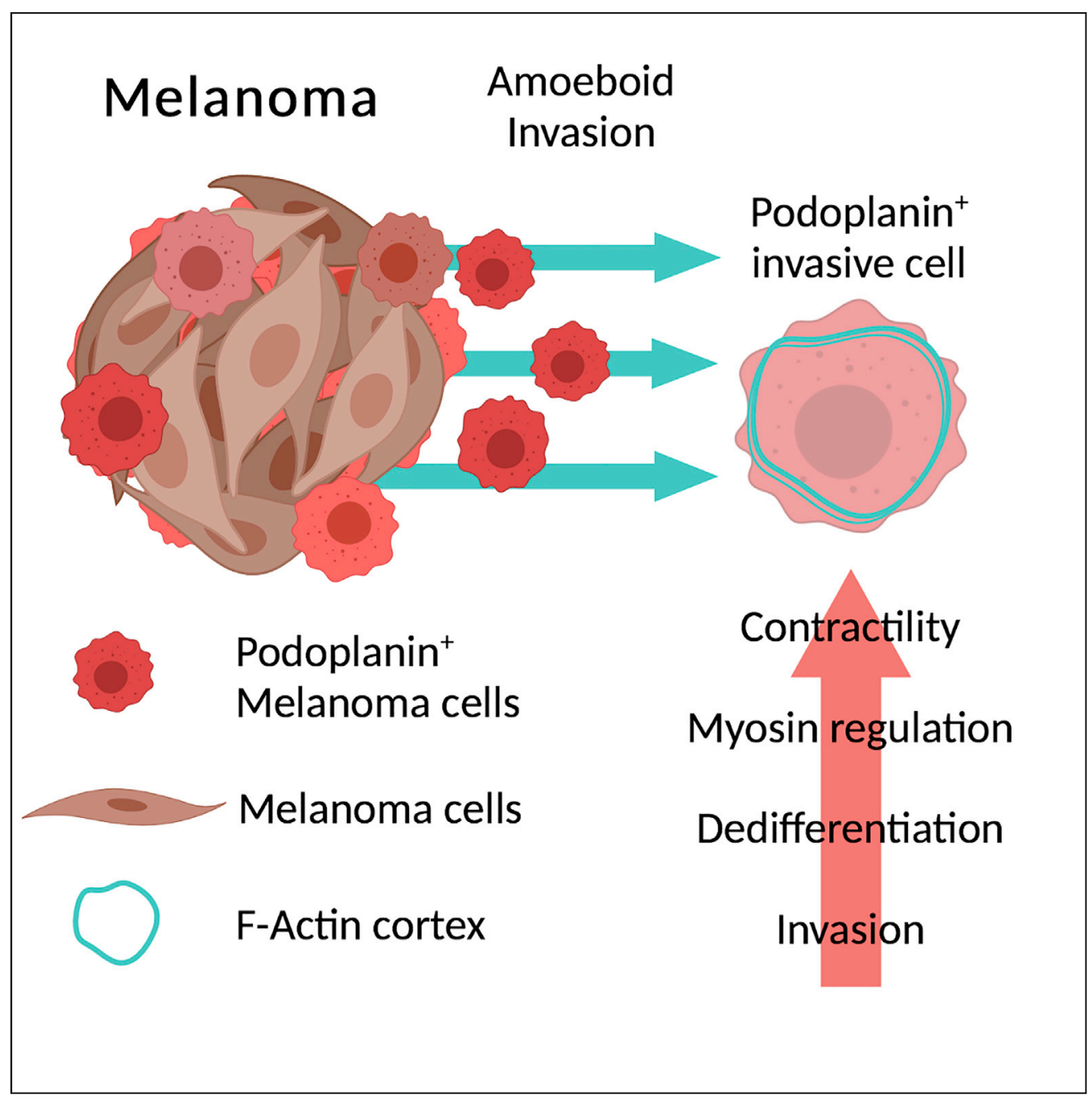

Charlotte M. de Winde, Samantha

L. George, Eva

Crosas-Molist, ...,

Víctor G. Martínez,

Victoria Sanz-

Moreno, Sophie E.

Acton

s.acton@ucl.ac.uk

Highlights

A dedifferentiated subset

of human melanomas

expresses high levels of

podoplanin

Amoeboid invasion is promoted by expression of the membrane

glycoprotein, podoplanin

Podoplanin induces phosphorylation of ERM and myosin to drive blebbing protrusions 


\title{
Article \\ Podoplanin drives dedifferentiation and amoeboid invasion of melanoma
}

\author{
Charlotte M. de Winde, ${ }^{1,4}$ Samantha L. George, ${ }^{1,2,4}$ Eva Crosas-Molist, ${ }^{2}$ Yukti Hari-Gupta, ${ }^{1}$ Abbey B. Arp, ${ }^{1}$ \\ Agnesska C. Benjamin, ${ }^{1}$ Lindsey J. Millward, ${ }^{1}$ Spyridon Makris, ${ }^{1}$ Alexander Carver, ${ }^{1}$ Valerio Imperatore, ${ }^{1,3}$ \\ Víctor G. Martínez, ${ }^{1}$ Victoria Sanz-Moreno, ${ }^{2}$ and Sophie E. Acton ${ }^{1,5, *}$
}

\section{SUMMARY}

Melanoma is an aggressive skin cancer developing from melanocytes, frequently resulting in metastatic disease. Melanoma cells utilize amoeboid migration as mode of local invasion. Amoeboid invasion is characterized by rounded cell morphology and high actomyosin contractility driven by Rho GTPase signalling. Migrastatic drugs targeting actin polymerization and contractility are therefore a promising treatment option for metastatic melanoma. To predict amoeboid invasion and metastatic potential, biomarkers functionally linked to contractility pathways are needed. The glycoprotein podoplanin drives actomyosin contractility in lymphoid fibroblasts and is overexpressed in many cancers. We show that podoplanin enhances amoeboid invasion in melanoma. Podoplanin expression in murine melanoma drives rounded cell morphology, increasing motility, and invasion in vivo. Podoplanin expression is increased in a subset of dedifferentiated human melanoma, and in vitro is sufficient to upregulate melanoma-associated marker Pou3f2/Brn2. Together, our data define podoplanin as a functional biomarker for dedifferentiated invasive melanoma and a promising migrastatic therapeutic target.

\section{INTRODUCTION}

Metastatic melanoma has a very poor prognosis, and there is a need for additional treatment options. Mutation of BRAF (V600E) causes hyperactivation of proliferation and survival signalling pathways (MAPK, ERK) driving melanoma progression (Gray-Schopfer et al., 2007). Combinations of BRAF inhibitors and immunotherapies targeting PD-1/PD-L1 show encouraging results; however, some patients develop resistance to treatment (Jenkins and Fisher, 2021). A novel alternative treatment strategy is to directly inhibit metastatic spread with migrastatic drugs, inhibiting actin polymerization and contractility mechanisms (Gandalovičová et al., 2017).

Metastasis is a multistep process, starting with local invasion of the surrounding tumor microenvironment and leading to systemic spread (Lambert et al., 2017). Cancer cells utilize and can switch between different modes of migration to invade through extracellular matrix and migrate to secondary sites (Pandya et al., 2017). Amoeboid migration is rapid and characterized by high actomyosin contractility driven by RhoA, Rho kinase (ROCK), and Myosin II signaling. Amoeboid invasive cells exhibit rounded cell morphology and continuous formation of protruding membrane blebs, allowing the cell to squeeze through the extracellular matrix (ECM) (Tozluoğlu et al., 2013) and reduce the requirement for proteolytic degradation of ECM (Pandya et al., 2017). Indeed, matrix metalloprotease inhibitors are ineffective in blocking amoeboid invasion (Wyckoff et al., 2006).

The transition to metastatic disease is a multi-faceted change in cancer cell state and not simply the initiation of cancer cell migration. Indeed, acquisition of invasive motility by melanoma cells has also been linked to a return to a more stem-like phenotype or dedifferentiation (Pinner et al., 2009; Hsiao and Fisher, 2014; Fane et al., 2019), closer to a trafficking melanoblast than a mature melanocyte. Moreover, high ROCK-Myosin II contractile signaling also drives changes in secretory pathways (Georgouli et al., 2019). Amoeboid-driven secretion of interleukin-1 alpha (IL- $1 \alpha$ ) and activation of NF- $\kappa \beta$ signaling results in macrophage differentiation which further promotes tumor growth and invasion (Georgouli et al., 2019).
${ }^{1}$ Stromal Immunology Group, MRC Laboratory for Molecular Cell Biology, University College London, Gower Street, London WC1E 6BT, UK

2Barts Cancer Institute, John Vane Science Building, Charterhouse Square, Queen Mary University of London, London EC1M 6BQ, UK

${ }^{3} \mathrm{Cell}$ Biogenesis and Tissue Regeneration Group, MRC Laboratory for Molecular Cell Biology, University College London, Gower Street, London WC1E 6BT, UK

${ }^{4}$ These authors contributed equally

${ }^{5}$ Lead contact

*Correspondence: s.acton@ucl.ac.uk

https://doi.org/10.1016/j.isci. 2021.102976 
A

$4 h$

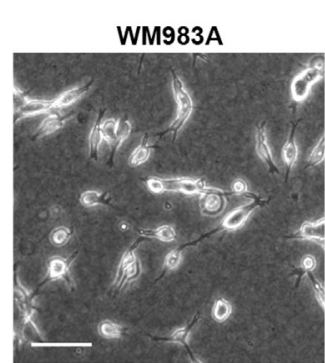

$24 h$

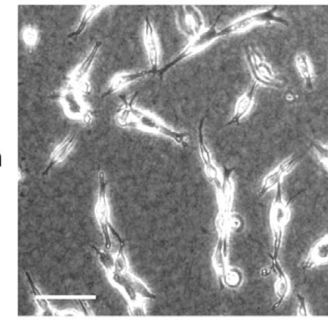

Brightfield
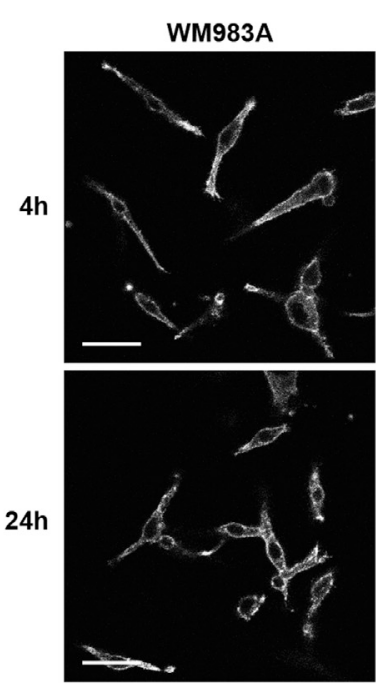

F-actin

D

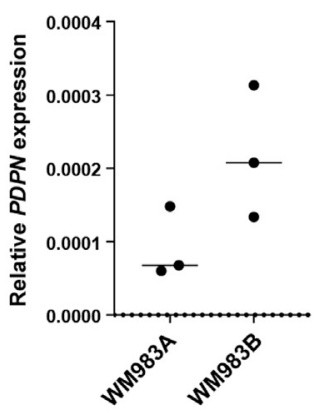

WM983B

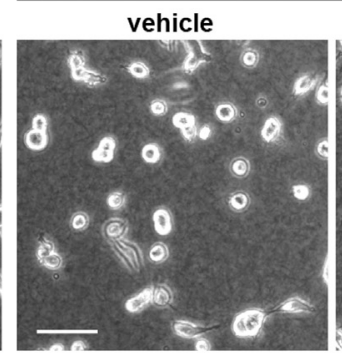

ROCK inhibitor
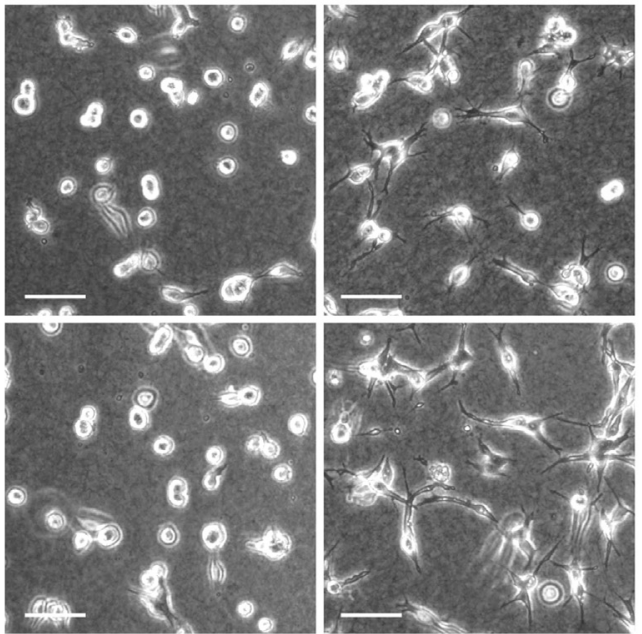

WM983B

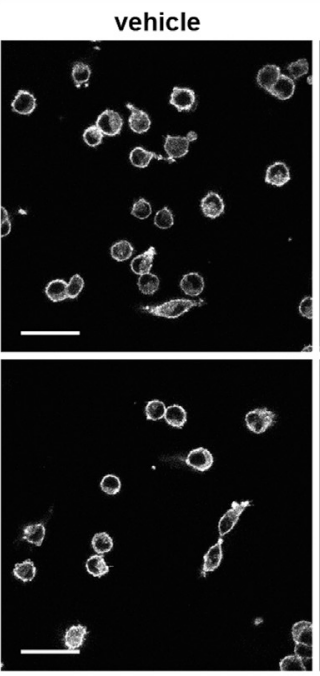

E

Kabbarah et al.

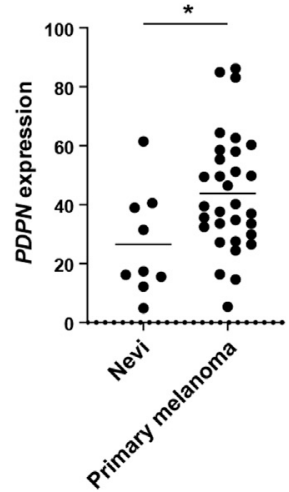

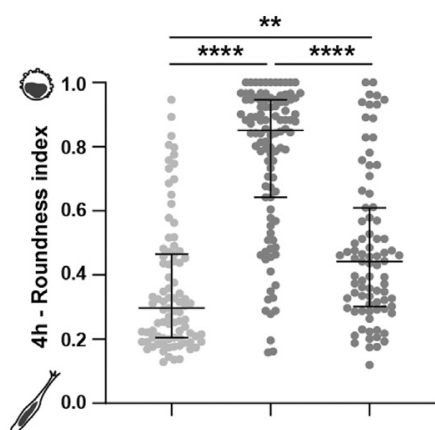

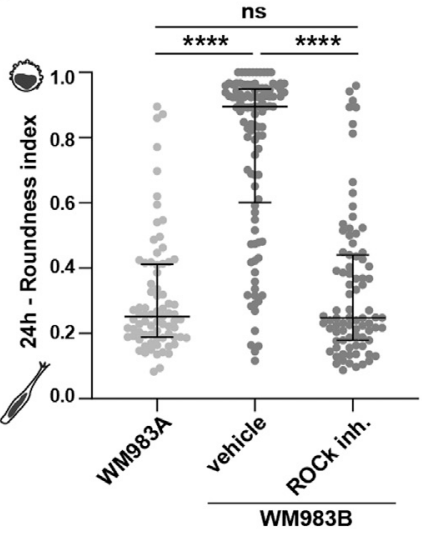

c

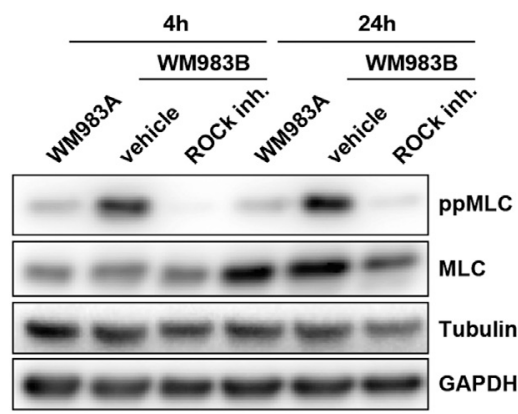

F

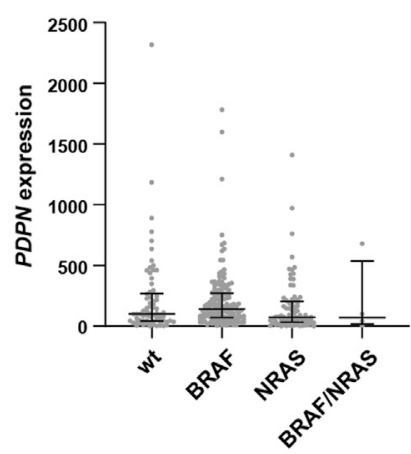


Figure 1. Podoplanin expression is increased in a subset of human melanomas

(A) Brightfield (upper panel) and immunofluorescence (lower panel) imaging of WM983A (left) and WM983B treated with vehicle (DMSO; middle) or ROCK inhibitor ( $1 \mu \mathrm{M}$ GSK269962A) for 4h (top) or 24h (bottom). Cells are stained for F-actin (white). The scale bars represent 100 (upper panel) or 40 (lower panel) microns.

(B) Cell morphology (roundness index) of WM983A (light grey) and WM983B (dark grey) cells as presented in panel (A) Kruskal-Wallis test with Dunn's multiple comparisons, ${ }^{* *} p=0.0036,{ }^{* * * *} p<0.0001$.

(C) Western blot analysis of ppMLC and MLC in WM983A (left) and WM983B treated with vehicle (DMSO; middle) or $1 \mu$ M ROCK inhibitor (GSK269962A) for 4h (top) or 24h (bottom). Tubulin and GAPDH are used as loading controls.

(D) Podoplanin (PDPN) mRNA expression in WM983A and WM983B cell lines measured by qPCR. PDPN mRNA expression is calculated as relative expression of the housekeeping gene $G A P D H\left(2^{\wedge}-\mathrm{Ct}(P D P N) / 2 \wedge-\mathrm{Ct}(G A P D H)\right)$. Data shown as mean with dots representing $\mathrm{n}=3$ biological replicates. Unpaired t test, two-tailed, $\mathrm{p}=0.1002$.

(E) Podoplanin (PDPN) mRNA expression in two datasets (Kabbarah et al. GSE46517 and Riker et al. GSE7553) of human melanoma and appropriate control tissues. Data shown as mean with dots representing melanoma tumors from individual patients. Kabbarah et al.: unpaired t test, two-tailed, * $\mathrm{p}=0.0250$; Riker et al:: Mann Whitney test, two-tailed, $\mathrm{p}=0.5052$.

(F) Podoplanin (PDPN) mRNA expression in human melanoma with BRAF and/or NRAS or no (wt) mutation in TCGA dataset (https://www.cancer.gov/tcga). Data are represented as median \pm interquartile range.

The cytoskeletal signaling cascades driving actomyosin contractility are well understood. However, there is also a need to identify potential biomarkers linked to contractility pathways, thereby predicting amoeboid invasion and metastatic progression. The membrane glycoprotein, podoplanin (also known as gp38, Aggrus, PA2.26, D2-40, T1 $\alpha$ ), drives actomyosin contractility in lymphoid fibroblasts (Acton et al., 2014) and is furthermore associated with myofibroblast phenotypes in inflammation and cancer (Quintanilla et al., 2019). We hypothesized that podoplanin also drives cytoskeletal contractility in cancer cells. Results of this study show that, podoplanin expression drives contractile amoeboid morphology in melanoma, thus triggering invasion and promoting metastasis.

\section{RESULTS}

\section{Podoplanin expression is increased in a subset of human melanomas}

Melanoma often acquires an amoeboid mode of invasion to metastasize, but the drivers of this conversion are incompletely understood. Podoplanin expression is known to be increased in many cancer types (Quintanilla et al., 2019) and drives actomyosin contractility through the activation of RhoA/C GTPases in fibroblasts (Acton et al., 2014). Because amoeboid cell migration and invasion requires high actomyosin contractility (Pandya et al., 2017), we asked whether podoplanin expression in melanoma could drive amoeboid invasion and promote metastasis. We first examined cell morphology and actomyosin contractility in the metastatic human melanoma cell line WM938B and its non-metastatic counterpart WM938A (Cantelli et al., 2015) (Figures 1A-1C, S1). On 2D rigid plastic, there are no clear morphological differences between WM938A and WM938B. However, when cultured on top of 3D deformable collagen-based matrices, WM983B cells acquired amoeboid morphology exhibiting a mostly rounded, contracted phenotype (roundness index $\geq 70 \%$ (Orgaz et al., 2014)), whereas WM983A cells exhibit a more spread, mesenchymal phenotype (Figure S1). Treatment with ROCK inhibitor (GSK269962A) reverted the acquired rounded phenotype of WM983B cells back to a mesenchymal morphology similar to WM983A cells (Figures 1A and 1B). These morphological differences observed between WM983B and WM938A cells are correlated with myosin light chain phosphorylation (ppMLC) and can also be reversed in the presence of ROCK inhibitor (Figure 1C). These data indicate that these metastatic melanoma cells acquire a rounded, amoeboid morphology through activation of actomyosin contractility.

This shift to contracted, rounded morphology correlates with an approximately 2.5-fold higher expression of PDPN mRNA in WM983B cells as measured by qPCR (Figure 1D). Examination of mRNA data in publicly available datasets from clinical primary melanoma biopsies confirmed that PDPN transcript levels are higher in a subset of melanoma samples than in controls (benign nevi or normal skin) (Figure 1E). However, in the TCGA dataset (https://www.cancer.gov/tcga), we found that podoplanin expression is independent of BRAF and/or NRAS mutation status, the two common mutations in human melanoma (Figure 1F). However, the cellular composition of these tissue samples will vary greatly, specifically the ratios of keratinocytes to melanocytes to fibroblastic stroma, especially between normal skin and melanoma. Therefore, we next directly examined podoplanin expression and function in a range of melanoma cell lines.

\section{Podoplanin promotes contractility in melanoma cells}

We examined podoplanin expression in mouse models of melanoma and found that — similarly to human melanoma (Figure 1F) - podoplanin surface protein expression is independent of Braf mutation status 
A

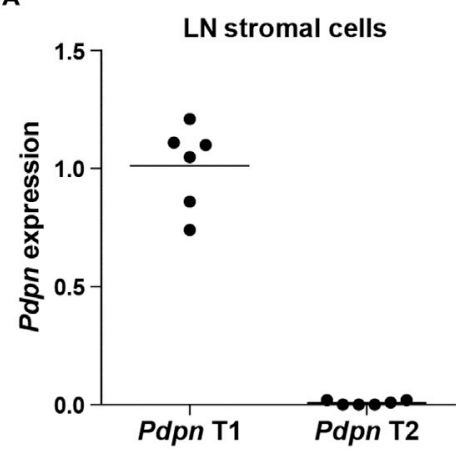

$\mathrm{B} 16 \mathrm{~F} 10$

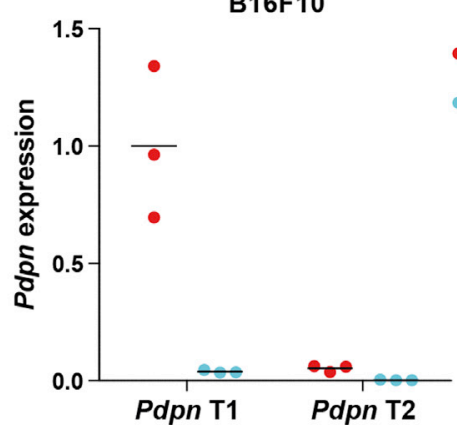

B

- $\mathrm{PDPN}^{+}$

- PDPN KO
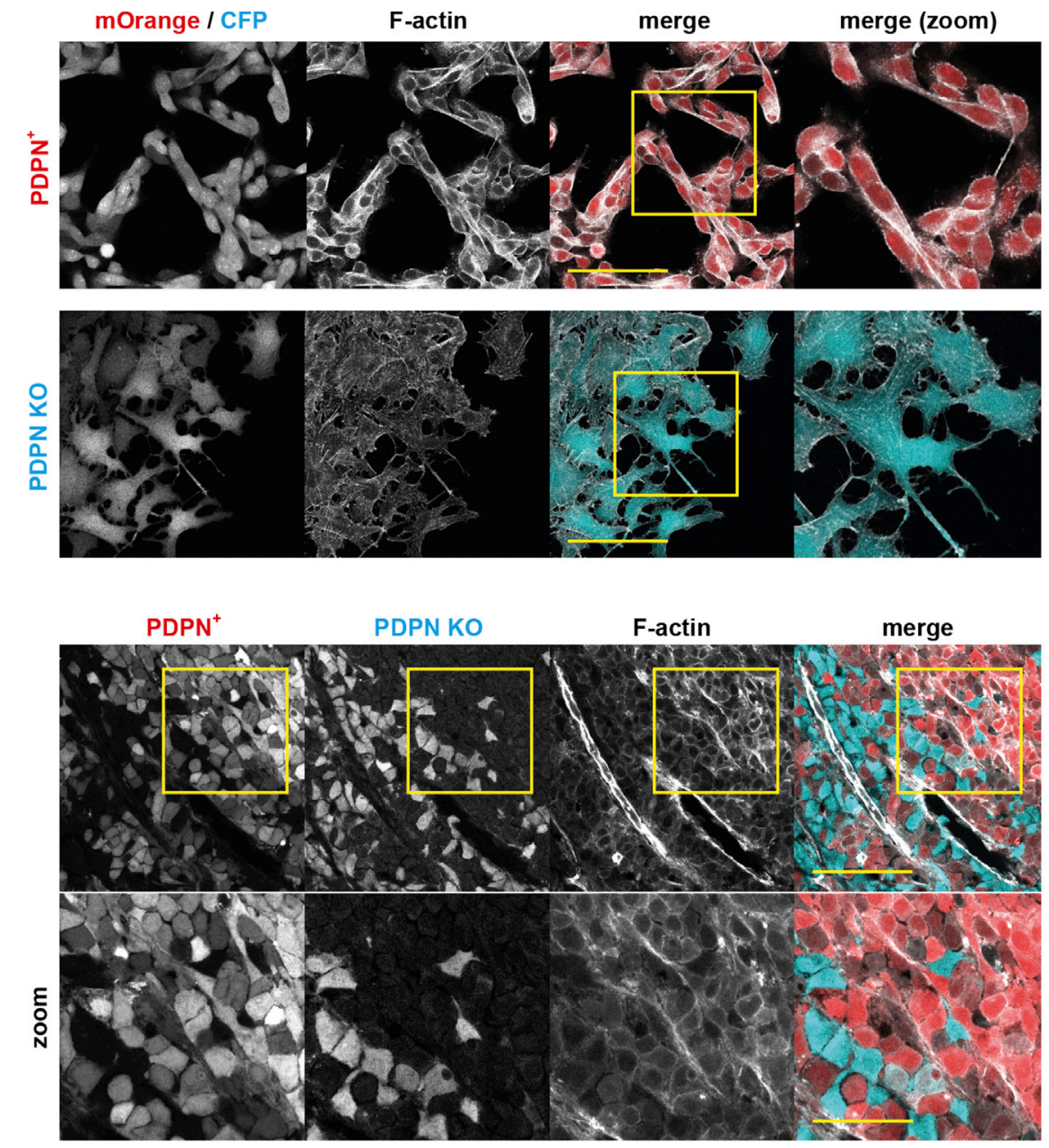
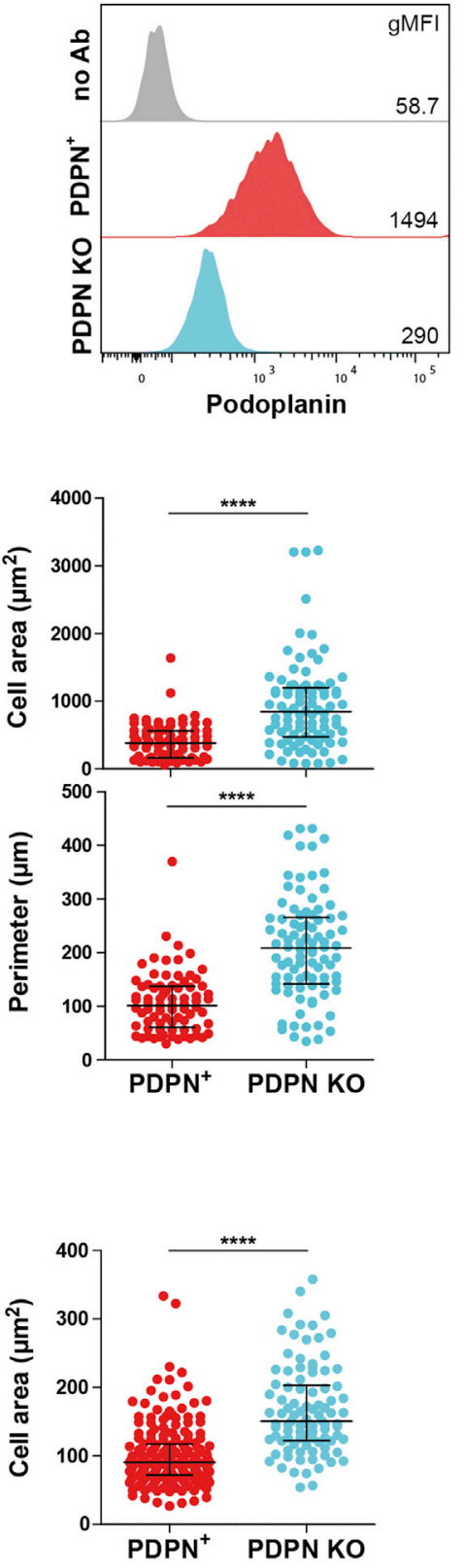

Figure 2. Podoplanin controls contractility of melanoma cells in vitro and in vivo

(A) Expression of podoplanin (Pdpn) mRNA transcript variant 1 (T1) and 2 (T2) in lymph node (LN) stromal cells cultured ex vivo for 3 days (left), and PDPN ${ }^{+}$ (red) and podoplanin knock-out (PDPN KO; blue) B16F10 murine melanoma cell lines (right). mRNA expression is calculated as fold change and normalized to Pdgfra (LN stromal cells) or Gapdh (B16F10) expression. Data shown as mean with dots representing $n=6$ (LN stromal cells) or $n=3$ (B16F10) biological replicates.

(B) Analysis of podoplanin surface expression in $\mathrm{PDPN}^{+}$(red) and PDPN KO (blue) B16F10 cell lines by flow cytometry. Cells not stained with antibody (no Ab; grey) are used as negative control. gMFI = geometric mean fluorescence intensity.

(C) Left: Immunofluorescence of F-actin (white) in $\mathrm{PDPN}^{+}$(top) and PDPN KO (bottom) B16F10 cell lines, labeled with respectively mOrange (red) or CFP (blue). Maximum z stack projections of representative images from $n=3$ biological replicates are shown. The scale bars represent 100 microns. Right: Cell 
Figure 2. Continued

area (in $\mu \mathrm{m}^{2}$; top) and perimeter (in $\mu \mathrm{m}$; bottom) of PDPN ${ }^{+}$(red) and PDPN KO (blue) B16F10 cells. Dots represent single cells. $\mathrm{n}=77-90$ cells collated from 3 biological replicates. Error bars represent median with interquartile range. Mann-Whitney test, two-tailed, ${ }^{\star \star \star \star} \mathrm{p}<0.0001$.

(D) Left: Immunofluorescence imaging of F-actin (white) in mixed PDPN ${ }^{+}$(red) and PDPN KO (blue) B16F10 tumor 9 days post-injection. The scale bars represent 100 (top) or 50 (bottom; zoom) microns. Right: Cell area of PDPN ${ }^{+}$(red) and PDPN KO (blue) B16F10 cells in the tumor. Dots represent single cells ( $n=97-197$ cells). Error bars represent median with interquartile range. Mann-Whitney test, two-tailed, ${ }^{\star \star \star \star} \mathrm{p}<0.0001$.

(Figure S2); Braf mutant cell lines range from podoplanin low (5555) to high expression (4434). B16F10 melanoma cells (Braf-V600E mutation negative) express intermediate levels of podoplanin protein (Figure S2). To directly test the contribution of podoplanin to melanoma morphology and invasion, we knocked out Pdpn (PDPN KO) in the metastatic cell line B16F10 using CRISPR/Cas9 technology. B16F10 cells predominantly express Pdpn transcript variant 1 , similarly to lymph node stromal cells where high endogenous podoplanin levels drive cytoskeletal contractility (Figure 2A). We confirmed that the guide RNAs successfully targeted podoplanin and both Pdpn mRNA, (Figure 2A) and the surface protein expression (Figure 2B) were reduced to background levels. PDPN ${ }^{+}$and PDPN KO B16F10 were then fluorescently labeled with either mOrange or CFP for direct comparison in mixed cell cultures. In in vitro cell culture, $\mathrm{PDPN}^{+}$cells grow in clusters with close cell to cell contacts (Figure 2C). Each PDPN ${ }^{+}$cell exhibits a rounded, contracted morphology, and intense F-actin staining is localized at the cell cortex (Figure 2C). PDPN KO cells are dramatically more spread and exhibit multiple protrusions as quantified by increased cell area and perimeter, respectively (Figure 2C), and no longer as clusters. PDPN KO B16F10 cells have lower F-actin intensity, and F-actin is arranged in filaments and puncta throughout the cell body (Figure 2C). Importantly, transfecting PDPN-CFP into PDPN KO cells restores the contracted cell morphology (Figure S3A). Similar to the human melanoma cells lines (Figure 1C), podoplanin-driven changes to B16F10 cells morphology were dependent on actomyosin contractility and treatment with ROCK inhibitor (GSK269962A) phenocopied PDPN KO cell morphology (Figure S3B).

We then asked whether podoplanin-driven contractility can control melanoma cell morphology in the tumor microenvironment in vivo. Podoplanin expression is maintained in vivo (Figure S4) and - similarly to our in vitro observations -, $\mathrm{PDPN}^{+}$cells have a smaller cross-sectional area than PDPN KO cells in the same tumour regions (Figure 2D). Moreover, cortical F-actin structures are also more prominent in $\mathrm{PDPN}^{+}$cells (Figure 2D). These results are consistent with the hypothesis that podoplanin intrinsically drives contractility of the actin cytoskeleton in melanoma cells to result in a rounded amoeboid-like cell morphology.

\section{Increased cell motility and invasion of podoplanin ${ }^{+}$melanoma cells in vivo}

Mice injected with a 1:1 mix of PDPN ${ }^{+}$(mOrange) and PDPN KO (CFP) generated tumors which often contained a higher proportion of PDPN ${ }^{+}$cells (Figures $3 \mathrm{~A}$ and S5), suggesting that podoplanin provides either a survival advantage or increased rate of proliferation. Consistent with this, we also find that $\mathrm{PDPN}^{+}$tumors grow much more rapidly in vivo than PDPN KO tumors (Figure S5A). However, neither podoplanin expression nor the fluorescence protein expressed had any effect on cell proliferation in vitro (Figure S5B). Upon examination of the cellular ratios of PDPN ${ }^{+}$(mOrange) vs PDPN KO (CFP) at the invasive front of mixed tumors, we observed a trend in 5/7 tumor sections that PDPN ${ }^{+}$B16F10 cells are overrepresented in melanoma tumor margins compared to the ratio of $\mathrm{PDPN}^{+}$:PDPN KO throughout the whole tumor (Figures $3 \mathrm{~B}$ and S6). Beyond the tumor boundary, the tumor capsule is frequently populated by host PDPN ${ }^{\text {hi }}$ cells likely to be fibroblasts. Within the fibroblastic-rich tumor capsule, the locally invasive tumor cells are almost exclusively PDPN ${ }^{+}$cells (Figure 3 C). These invasive PDPN ${ }^{+}$tumor cells which have locally invaded beyond the tumor boundary have a rounded morphology and are exclusively observed invading as single cells (Figure 3 C). Local invasion of individual cancer cells through the matrix requires a balance of proteolysis and high cytoskeletal contractility, permitting amoeboid invasion. The proteolytic activity of $\mathrm{PDPN}^{+}$and PDPN KO melanoma cells is comparable (Figure S7) indicating that podoplanin is not promoting local invasion via increased proteolysis in this model, but could instead be promoting amoeboid invasion. We next sought to ask whether overexpression of podoplanin (PDPN-CFP) in PDPN ${ }^{10} 5555$ melanoma cells (Figure S2A) is sufficient to enhance melanoma motility in vivo. We overexpressed PDPN-CFP in a subset of mCherry-labeled 5555 cells. More than $60 \%$ of 5555 -mCherry cells which overexpress PDPN-CFP move as single cells with largely rounded morphology and rapid changing cell shapes (Figures 3D-3E and Video S1). A close examination and surface rendering of individual motile PDPN-CFP expressing cells shows multiple bleb-like protrusions, consistent with an amoeboid phenotype, suggesting that overexpression of podoplanin can promote amoeboid motility in vivo. 

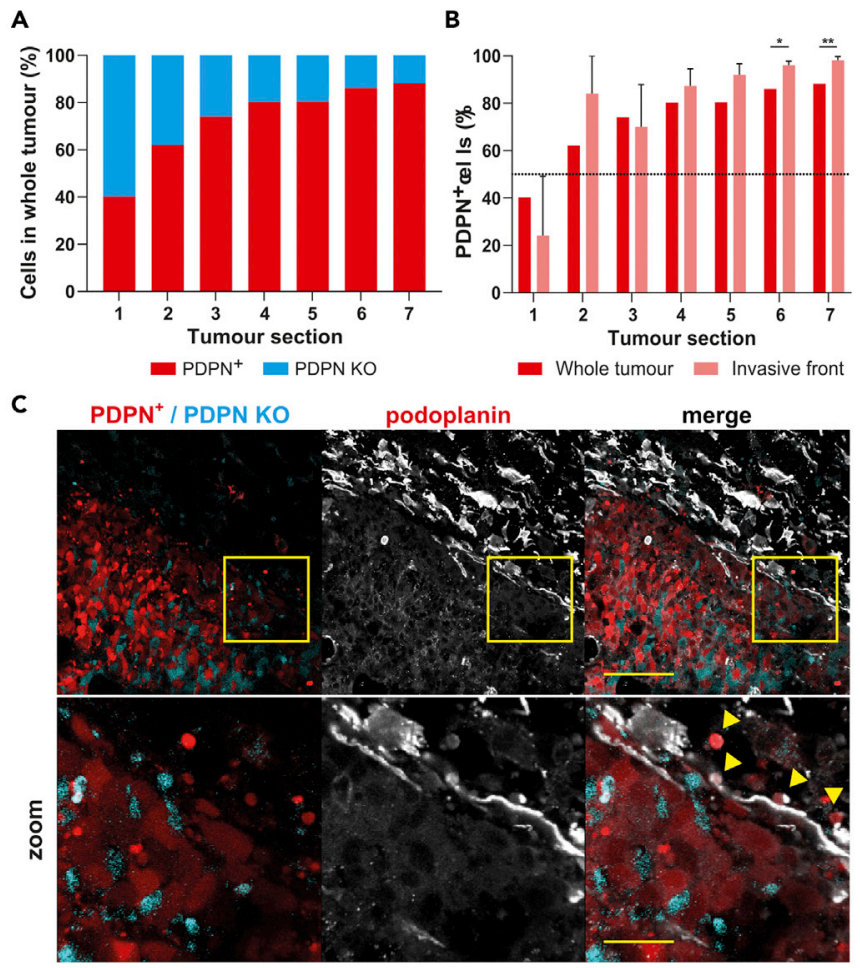

D
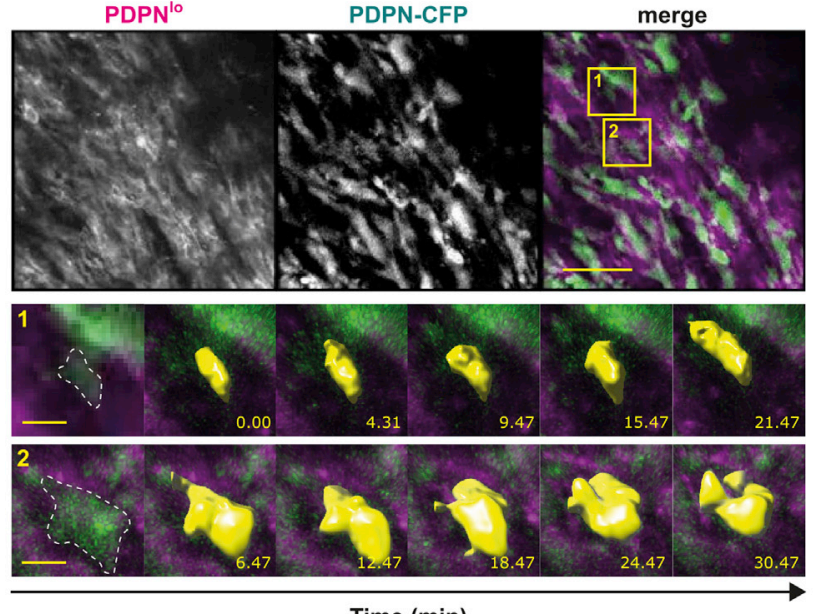

Time (min)

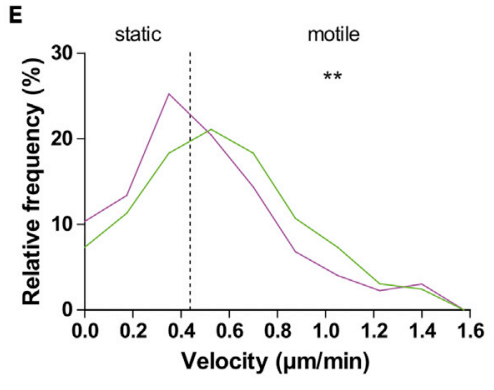

— 5555-mCherry

- PDPN-CFP

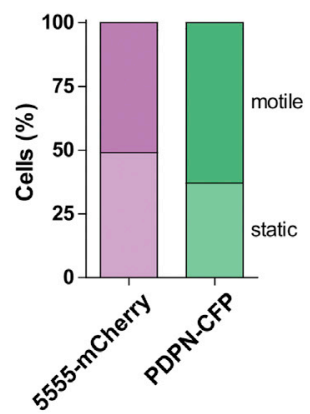


Figure 3. Podoplanin dependent transition to amoeboid dissemination in melanoma cells

(A) Proportions of PDPN ${ }^{+}$(red) and PDPN KO (cyan) cell populations within mixed B16F10 tumors. 7 individual sections across 2 different tumors were analyzed using QuPath software (Figure S5).

(B) Enrichment graph showing proportions of PDPN ${ }^{+}$B16F10 cells in the invasive front (IF) compared to overall proportions within mixed PDPN ${ }^{+} /$PDPN KO whole tumors. Data is shown as median with interquartile range. Multiple t-tests, ${ }^{\star} \mathrm{p}=0.024,{ }^{\star *} \mathrm{p}=0.003$

(C) Immunofluorescence imaging of podoplanin (white) in mixed PDPN ${ }^{+}$(red) and PDPN KO (blue) B16F10 tumor 9 days post-injection. Arrow heads indicate disseminated PDPN ${ }^{+}$B16F10 cells. The scale bars represent 100 (top) or 50 (bottom; zoom) microns.

(D) Time-lapse imaging of mixed PDPN ${ }^{10}$ (mCherry-labeled; magenta) and PDPN-CFP (green) transfected 5555 murine melanoma tumor (see also Video S1). Dashed line indicates PDPN-CFP 5555 -mCherry cell tracked over time, with zooms

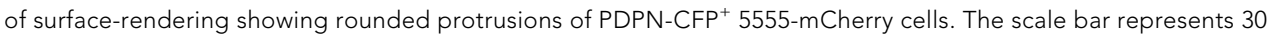
microns (top) 5 microns (lower panels 1 and 2). Time of each frame indicated in minutes.

(E) Left: Velocity of PDPN ${ }^{\text {lo }}$ (magenta) and PDPN-CFP (green) transfected 5555-mCherry cells in vivo. Mann Whitney test, two-tailed, ${ }^{\star \star} \mathrm{P}=0.0013$. Right: Percentage of static or motile PDPN ${ }^{\text {lo }}$ (magenta) and PDPN-CFP (green) transfected 5555-mCherry cells.

\section{Overexpression of podoplanin induces amoeboid blebbing of melanoma cells}

Amoeboid migration is characterized by high actomyosin contractility and membrane blebbing to induce a path-seeking mode of invasion for metastasizing cells to escape a primary tumour (Tozluoğlu et al., 2013). We undertook high-resolution live confocal imaging of 5555 melanoma cells that were overexpressing PDPN-CFP, to definitively determine whether PDPN expression induces amoeboid blebbing in melanoma cells. PDPN-CFP-expressing 5555 cells exhibit large and dynamic membrane blebs (Figure 4A). We compared the localization of PDPN-CFP and LifeAct in membrane blebs during bleb retraction. Interestingly, podoplanin was localized to the plasma membrane, ahead of reassembly of the actin cortex. LifeAct is observed predominantly in the cell cortex and is at lower concentration in the bleb (Figures 4A and 4B). In contrast, we find that podoplanin is enriched at the plasma membrane throughout blebbing and is increased in intensity in the plasma membrane, as the bleb retracts (Figures $4 \mathrm{~A}$ and $4 \mathrm{~B}$ ).

We then used an alternative model to induce podoplanin expression using a doxycycline-inducible construct. In 5555 melanoma cells seeded in a collagen matrix, we confirm that induction of podoplanin expression correlates with higher levels of both phosphorylated MLC and ezrin, radixin, and moesin (ERM) family proteins (Figures 4C and 4D), indicating higher actomyosin contractility and membrane-cortex attachment. In fibroblasts, podoplanin has been shown to regulate pERM to induce cell contractility and cell rounding, and indeed a dominant negative mutant of ezrin is able to block podoplanin-dependent cell rounding (Acton et al., 2014). Here we show that podoplanin also upregulates pERM in melanoma cell lines (Figure 4C), indicating that PERM is regulated by podoplanin in both of these different cell types. Importantly, we observe this positive correlation between PDPN:PMLC and PDPN:pERM even in cells expressing relatively low levels of podoplanin (Figure 4C). 5555 cells with high induction of podoplanin expression also exhibit membrane blebs (Figure 4D). Membrane blebs can also be observed in apoptotic cells; therefore, to determine whether the blebs we observe are linked to protrusion formation or due to apoptosis, we also undertook an apoptosis assay. 5555 melanoma cells expressing the inducible podoplanin construct were treated with doxycycline for either 1 day or 5 days (Figure 4E). After 1 day, the majority of cells (75\%) expressed podoplanin; after 5 days, the majority of cells (92\%) expressed podoplanin and the expression levels were higher than at day 1 (Figure 4E). We directly compared the expression of apoptosis markers Annexin $V$ and 7AAD in podoplanin ${ }^{+}$versus podoplanin 5555 cells from the same time points (Figure 4F). We quantified very few apoptotic cells in any sample ( $>5 \%)$, but importantly, no difference in apoptosis upon induction of podoplanin expression, even at high levels of expression as seen after 5 days of doxycycline induction (Figures $4 \mathrm{E}$ and $4 \mathrm{~F}$ ). Further, close examination of the nuclei of blebbing and contracted podoplanin-induced 5555 cells shows no condensation or fragmentation of DNA (Figure 4D), leading us to rule out apoptosis and to conclude that podoplanin-induced blebbing is consistent with the acquisition of amoeboid morphology.

\section{Podoplanin expression drives dedifferentiation of melanoma}

Acquisition of an invasive motile phenotype is linked with dedifferentiation of melanoma, marked by loss of melanocyte functions such as pigmentation (Hsiao and Fisher, 2014). We observe that PDPN KO B16F10 tumors are more pigmented compared to $\mathrm{PDPN}^{+}$tumors (Figure $5 \mathrm{~A}$ ). This altered pigmentation is cell intrinsic and maintained in mixed in vitro cultures (Figures $5 \mathrm{~B}$ and $5 \mathrm{C}$ ), suggesting that knock-out of 


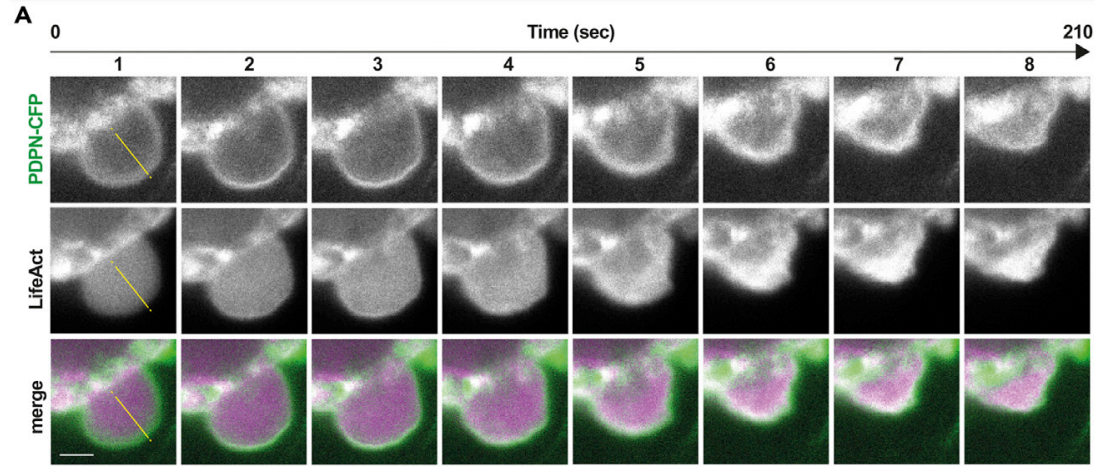

B
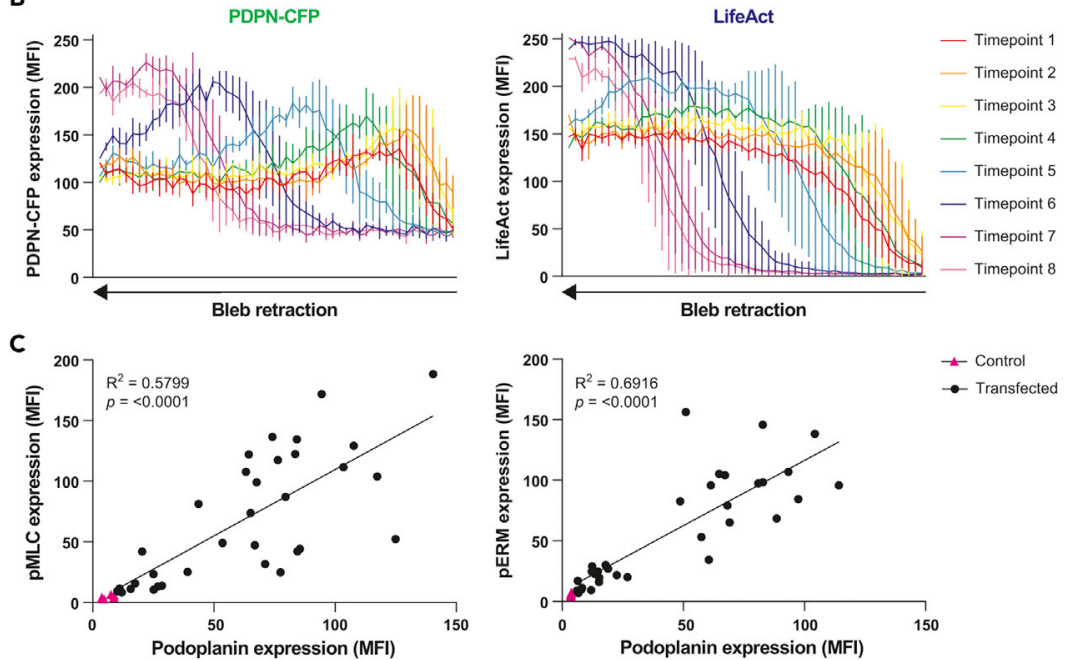

D
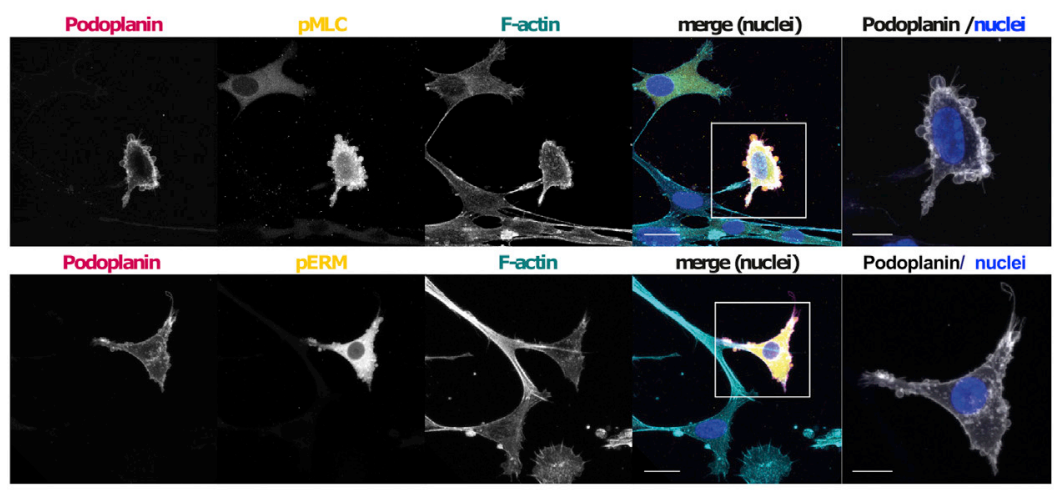

E
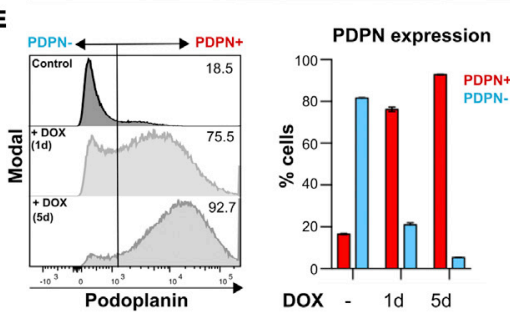

$\mathbf{F}$
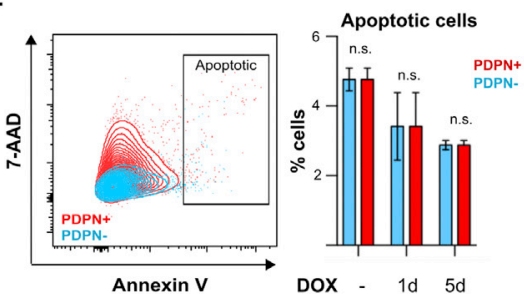

Figure 4. Overexpression of podoplanin drives amoeboid blebbing

(A) Time-lapse imaging of 5555 cells transfected with PDPN-CFP (magenta) and LifeAct (green). The yellow dashed line is representative for the line plot used to quantify PDPN-CFP and LifeAct intensity as shown in panel (B) The scale bar represents 2 microns. 
Figure 4. Continued

(B) PDPN-CFP (left) and LifeAct (right) expression (as mean fluorescence intensity (MFI)) at timepoints indicated in panel (A) Expression is measured at three lines (length = 3.35 microns; example in panel A (yellow dashed line)) through the bleb. Analysis of one representative bleb is shown. Data shown as mean \pm SD

(C) Podoplanin and pMLC (left) or pERM (right) expression (as mean fluorescence intensity (MFI) in 5555 untreated cells (control; pink triangles), or 5555 cells treated with DOX (to induce podoplanin expression) and transfected with pMLC or pERM (transfected; black dots).

(D) Podoplanin (magenta), pMLC (top) or pERM (bottom) (yellow), F-actin (cyan) expression in 5555 cells treated with DOX (to induce podoplanin expression) and transfected with pMLC or pERM. Nuclei are stained with DAPI (blue). The scale bars represent 20 and 10 (zoom) microns.

(E) Representative histograms and quantification of podoplanin surface expression following induction with doxycycline. Data shown as mean $\pm \mathrm{SD}, \mathrm{N}=3$ biological replicates.

(F and E) Representative contour plot and quantification of percentages of apoptotic cells following podoplanin induction as shown in (E) Data shown as mean $\pm S D, N=3$ biological replicates.

podoplanin expression restores pigmentation, a characteristic of non-invasive differentiated melanocytes (Hsiao and Fisher, 2014).

To explore whether podoplanin expression altered gene expression linked to melanoma differentiation, we examined mRNA expression in human primary melanoma patient samples and cell lines. When clustered based on PDPN expression, we found that a subset of melanoma expressed high levels of podoplanin (Figures 1D and 5E). We found that the PDPN ${ }^{\text {hi }}$ samples expressed lower levels of key melanocyte genes, including tyrosinse (TYR) and premelanosome protein (PMEL) (Figure 5D). This is consistent with changes in pigmentation we observe in $\mathrm{PDPN}^{+}$vs PDPN KO melanoma orthotopic tumors and cell lines (Figures 5A-5C). Consistent with our analysis of the TCGA dataset (Figure 1F), podoplanin expression in these patient samples was not correlated with either BRAF or NRAS mutation status (Figure 5D). Next, we directly tested the impact of podoplanin on melanocyte gene expression. Transcription of melanocyte-associated markers required for pigmentation, Mlana, and Tyr (Hsiao and Fisher, 2014) are also downregulated in $\mathrm{PDPN}^{+}$B16F10 mouse melanoma cell line compared to PDPN KO controls, whereas the melanoma-associated genes Mitfand Pou3f2 are upregulated (Figure 5E). Comparisons of PDPN ${ }^{\text {hi }}$ vs. PDPN KO cells partially correlate with the patient data (Figures 5D and 5E), suggesting that podoplanin is not the single driver of an invasive melanoma, but may contribute to this phenotype. We also tested the impact of podoplanin on the expression of dedifferentiation markers at the protein level (Figure 5F). Despite reduced mRNA transcription, Melan-A and tyrosinase protein expression is not reduced in PDPN ${ }^{+}$cells. However, podoplanin is clearly impacting pigmentation pathways in these cell lines (Figure 5A), and the mechanisms impacting pigmentation pathways in melanoma would require further investigation. Pou $3 f 2$ encodes the transcription factor Brn2, a major regulator of melanoma phenotype switching, and is directly linked with dedifferentiation and acquisition of motility (Pinner et al., 2009; Fane et al., 2019). Both mRNA and protein expression of Pou3f2/Brn2 are positively correlated to podoplanin levels in these cell lines (Figures 5E and 5F), matching a subset of PDPN ${ }^{+}$patient-derived cell lines (Figure 5D). Overall, these data provide evidence that high podoplanin expression is linked with a more dedifferentiated and invasive subset of melanoma, and expression of a key melanoma regulator, Pou3f2/Brn2, is directly sensitive to podoplanin expression.

\section{DISCUSSION}

It is known that cancer cells can utilize amoeboid migration for local invasion and initiation of metastasis, and the cytoskeletal rearrangements controlling this mode of migration are well studied. However, how these signaling pathways become hyper activated are not fully understood, and we lack druggable molecular targets to inhibit amoeboid invasion. Here, we show that expression of podoplanin, a known driver of actomyosin contractility in fibroblasts, is upregulated in a subset of melanoma patients. We show that podoplanin is able to drive high cytoskeletal contractility in melanoma cells, promoting an invasive amoeboid phenotype. In agreement with our presented data, high podoplanin expression has been observed at the invasive front of different cancer types (Quintanilla et al., 2019), where individual invading amoeboid cells are also observed (Georgouli et al., 2019; Cantelli et al., 2015; Sanz-Moreno et al., 2008).

Pro-inflammatory cytokines have been linked to increased actomyosin contractility and amoeboid invasion in stroma and tumour cells (Georgouli et al., 2019; Sanz-Moreno et al., 2011). In addition, cytokine signaling in the tumor microenvironment is one pathway known to induce aberrant expression of podoplanin (Quintanilla et al., 2019). Podoplanin expression can also be induced by the activity of oncogenes such 
A

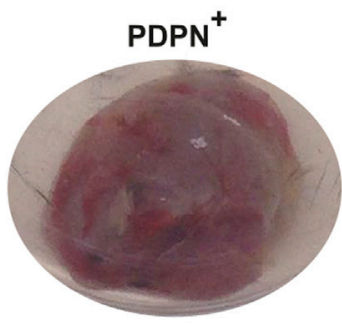

C

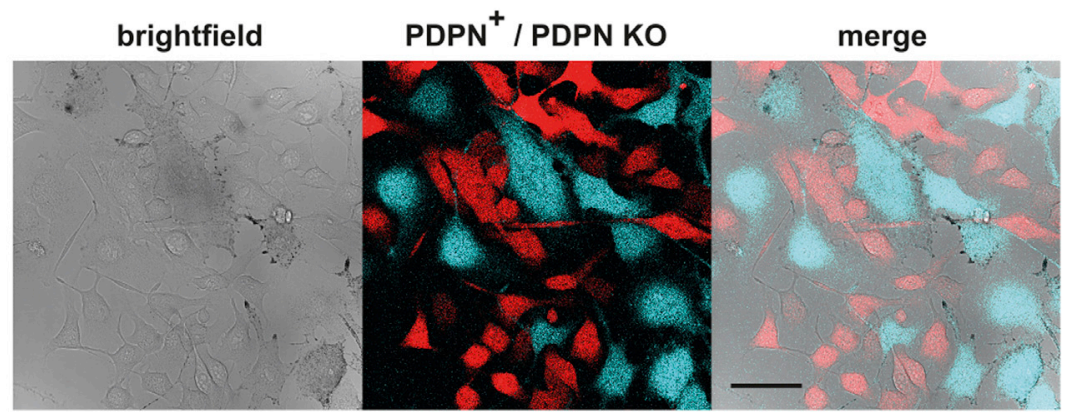

B

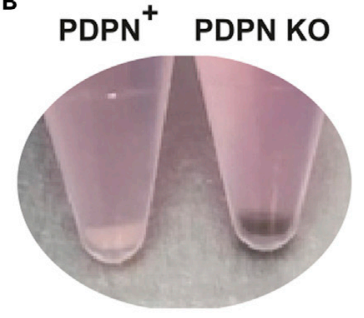

D

\section{Riker et al.}

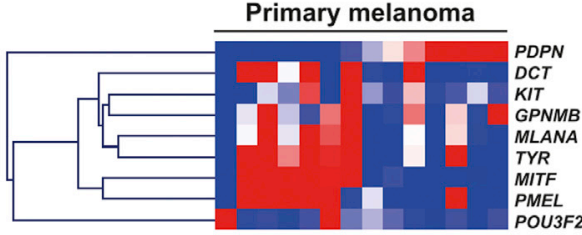

Mannheim cohort

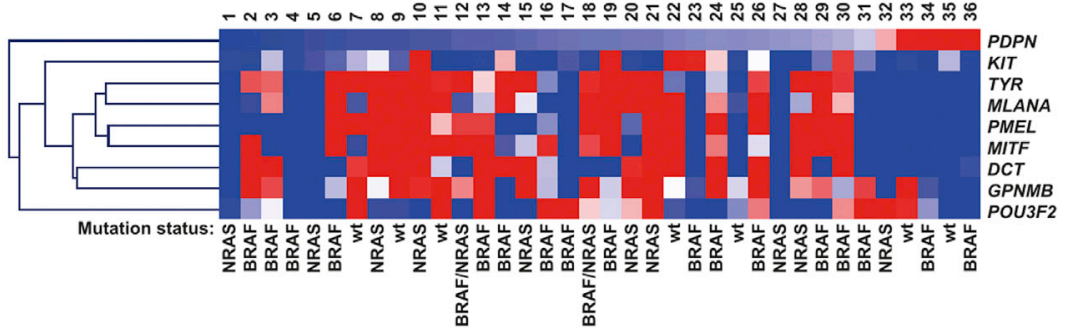

E

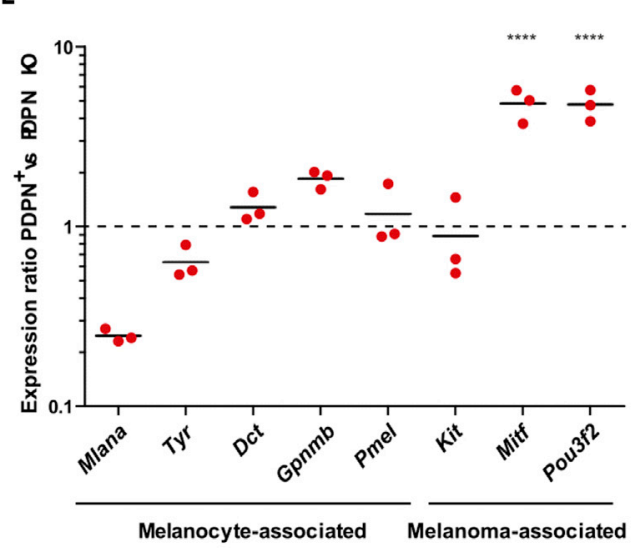

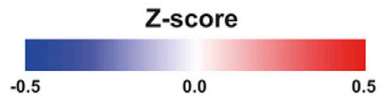

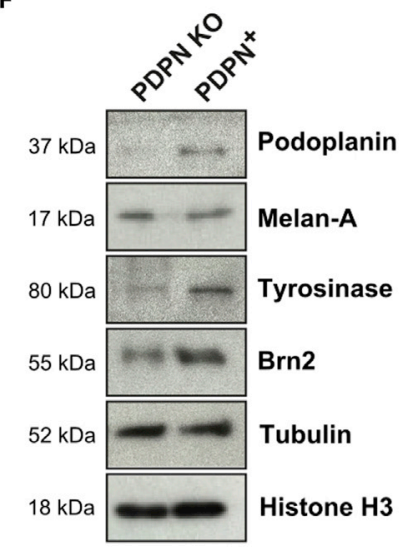


Figure 5. Loss of podoplanin restores pigmentation and melanocyte differentiation

(A and B) Tumors (A) and cells pellets (B) of PDPN ${ }^{+}$and PDPN KO B16F10 cell lines.

(C) Imaging of pigmentation (brightfield; left) of PDPN ${ }^{+}$and PDPN KO B16F10 cell lines, labeled with mOrange (red) or

CFP (blue) respectively (middle). The scale bar represents 50 microns.

(D) Heatmaps showing expression (Z score) of podoplanin (PDPN) and eight dedifferentiation-associated genes in

datasets of primary tumor samples of melanoma patients (top; Riker et al. GSE7553) and metastatic melanoma cultures (bottom; Mannheim cohort, GSE4843; from Hoek et al., 2006). For the Mannheim cohort, each number indicates a separate metastatic melanoma culture, and the BRAF and/or NRAS mutation status is indicated for each. (E) mRNA expression of five melanocyte-associated (Mlana/Mart-1 ( $p=0.2045), \operatorname{Tyr}(p=0.9276)$, Dct $(p=0.9859)$, Gpnmb $(p=0.1308)$, Pmel ( $p=0.9994)$; left) and three invasion-associated (Kit $(p>0.9999)$, Mitf (**** $p<0.0001)$, Pou3f2 (encodes $\mathrm{Brn2}$; ${ }^{* * \star} \mathrm{p}<0.0001$; right) genes in PDPN ${ }^{+}$B16F10 cells. mRNA expression is calculated as fold change of Gapdh expression and normalized to expression in PDPN KO B16F10 cells (set at 1 as indicated by dashed line). Data shown as mean with dots representing $n=3$ biological replicates. Two-way ANOVA with Sidak's multiple comparisons; $p$ values indicated above.

(F) Western blot analysis of Podoplanin, Melan-A, Tyrosinase and Brn2 (encoded by Pou3f2 gene) in PDPN KO and PDPN ${ }^{+}$ B16F10 cells. Tubulin and histone $\mathrm{H} 3$ are used as loading controls.

as Src kinase (Shen et al., 2010) — and furthermore the transcription factor AP-1 — which directly binds the podoplanin promotor in malignant keratinocytes resulting in increased podoplanin expression in skin tumors (Durchdewald et al., 2008). We have recently shown that binding of the podoplanin binding partner, C-type lectin-like receptor 2 (CLEC-2), in addition to inhibiting podoplanin-driven contractility (Acton et al., 2014), also upregulates podoplanin expression (de Winde et al., 2021). CLEC-2 is highly expressed on platelets, which may leak into the tumor via poorly functioning blood vessels. This interaction may further upregulate podoplanin expression on tumor cells, leading to a more amoeboid and invasive phenotype.

It was already known that podoplanin expression was correlated with poor prognosis and higher incidence of metastatic disease (Quintanilla et al., 2019), but the mechanisms were not fully understood. We now show that podoplanin is a driver of amoeboid invasion, and furthermore we link podoplanin directly to melanoma dedifferentiation. The amoeboid cell state supports both invasion and tumor cell 'stemness' (Rodriguez-Hernandez et al., 2020). Our data supports that podoplanin expression drives a wide-ranging reprogramming of melanoma towards a dedifferentiated, invasive phenotype with higher capacity for tumor initiation or proliferation. We predict that targeting podoplanin to block downstream signaling in melanoma would reverse dedifferentiation, and as such would inhibit invasion and metastasis. A potential strategy could be to design small-molecules to mimic CLEC-2 binding to podoplanin. Podoplanin has potential as a diagnostic biomarker for dedifferentiated melanoma, as well as migrastatic drug target, which could be used in combination with current treatments for melanoma.

\section{Limitations of the study}

We show here that podoplanin expression level is linked with a dedifferentiated phenotype for melanoma, and that changing podoplanin expression is sufficient to alter transcription of genes linked with differentiation status. However, at this time, it is not known how membrane expression of podoplanin is mechanistically linked to transcriptional regulation, and to date, no studies of podoplanin-dependent transcription have been undertaken. This will be an interesting direction for future research, and the impact of podoplanin on transcriptional regulation may be broader than melanoma, because podoplanin is widely expressed on many varied cell types.

Melanoma cells have been broadly categorized into melanocytic proliferative and dedifferentiated mesenchymal-like invasive phenotypes, which cells can switch between. Here we show that podoplanin-expressing melanoma are invasive and dedifferentiated but that they acquire an amoeboid phenotype rather than mesenchymal. Therefore the podoplanin ${ }^{+}$subset of melanoma that we identify do not easily fit into either of these broad categories. Further investigation would be required to understand how podoplanin ${ }^{+}$mel- $^{-}$ anoma fits within the current understanding of melanoma subtypes.

\section{STAR $\star$ METHODS}

Detailed methods are provided in the online version of this paper and include the following:

- KEY RESOURCES TABLE

- RESOURCE AVAILABILITY 
O Lead contact

O Materials availability

O Data and code availability

- EXPERIMENTAL MODEL AND SUBJECT DETAILS

O Mice

- METHOD DETAILS

○ Animal procedures

O In vitro cell proliferation assay

O In vitro proteolysis assay

Immunofluorescence imaging \& analysis

- Flow cytometry

Apoptosis assay

O RNA isolation and quantitative RT-PCR analysis

O Western blotting

O Melanoma patient and cell lines mRNA database analysis

- QUANTIFICATION AND STATISTICAL ANALYSIS

\section{SUPPLEMENTAL INFORMATION}

Supplemental information can be found online at https://doi.org/10.1016/j.isci.2021.102976.

\section{ACKNOWLEDGMENTS}

We thank Prof R. Marais for providing 5555 parental melanoma cell lines. This work is funded by Cancer Research UK (Career development fellowship CRUK-A19763 to S.E.A.; C33043/A12065 and C33043/ A24478 to V.S.-M.), Rubicon Postdoctoral fellowship from the Dutch Research Council (NWO; 019.162LW.004; to C.M.d.W.), Medical Research Council (MC-U12266B), Barts Charity and Fundación Ramón Areces.

\section{AUTHOR CONTRIBUTIONS}

C.M.d.W. and S.E.A. designed and supervised the study and co-wrote the manuscript. C.M.d.W, S.L.G, A.B.A, A.C.B., S.M., E.C-M., Y.H-G., A.C., V.I., and V.G.M., performed experiments, E.C-M. performed bioinformatics analysis. C.M.d.W, V.S-M., and S.E.A edited the manuscript.

\section{DECLARATIONS OF INTEREST}

The authors declare no competing financial interests.

Received: September 16, 2020

Revised: May 12, 2021

Accepted: August 10, 2021

Published: September 24, 2021

\section{REFERENCES}

Acton, S.E., Farrugia, A.J., Astarita, J.L., MourãoSá, D., Jenkins, R.P., Nye, E., Hooper, S., van Blijswijk, J., Rogers, N.C., Snelgrove, K.J., et al. (2014). Dendritic cells control fibroblastic reticular network tension and lymph node expansion. Nature 514, 498-502. https://doi.org/10.1038/ nature 13814

Cantelli, G., Orgaz, J.L., Rodriguez-Hernandez, I., Karagiannis, P., Maiques, O., Matias-Guiu, X., Nestle, F.O., Marti, R.M., Karagiannis, S.N., and Sanz-Moreno, V. (2015). TGF- $\beta$-Induced transcription sustains amoeboid melanoma migration and dissemination. Curr. Biol. 25, 2899-2914. https://doi.org/10.1016/j.cub.2015 09.054.

Durchdewald, M., Guinea-Viniegra, J., Haag, D., Riehl, A., Lichter, P., Hahn, M., Wagner, E.F.,
Angel, P., and Hess, J. (2008). Podoplanin is a novel fos target gene in skin carcinogenesis. Cancer Res. 68, 6877-6883. https://doi.org/10. 1158/0008-5472.CAN-08-0299.

Fane, M.E., Chhabra, Y., Smith, A.G., and Sturm, R.A. (2019). BRN 2, a POU erful driver of melanoma phenotype switching and metastasis. Pigment Cell Melanoma Res 32,9-24. https://doi. org/10.1111/pcmr.12710.

Gandalovičová, A., Rosel, D., Fernandes, M., Veselý, P., Heneberg, P., Cermák, V., Petruželka, L., Kumar, S., Sanz-Moreno, V., and Brábek, J. (2017). Migrastatics-anti-metastatic and antiinvasion drugs: promises and challenges. Trends Cancer 3, 391-406. https://doi.org/10.1016/j. trecan.2017.04.008.
Georgouli, M., Herraiz, C., Crosas-Molist, E., Fanshawe, B., Maiques, O., Perdrix, A., Pandya, P., Rodriguez-Hernandez, I., Ilieva, K.M., Cantelli, G., et al. (2019). Regional activation of myosin II in cancer cells drives tumor progression via a secretory cross-talk with the immune microenvironment. Cell 176, 757-774.e23. https://doi.org/10.1016/j.cell.2018.12.038.

Gray-Schopfer, V., Wellbrock, C., and Marais, R. (2007). Melanoma biology and new targeted therapy. Nature 445, 851-857. https://doi.org/10. 1038/nature05661.

Hoek, K.S., Schlegel, N.C., Brafford, P., Sucker, A., Ugurel, S., Kumar, R., Weber, B.L., Nathanson, K.L., Phillips, D.J., and Herlyn, M. (2006). Metastatic potential of melanomas defined by specific gene expression profiles with no BRAF 
signature. Pigment Cell Res 19, 290-302. https:// doi.org/10.1111/j.1600-0749.2006.00322.x.

Hsiao, J.J., and Fisher, D.E. (2014). The roles of microphthalmia-associated transcription factor and pigmentation in melanoma. Arch. Biochem. Biophys. 563, 28-34. https://doi.org/10.1016/j. abb.2014.07.019.

Jenkins, R.W., and Fisher, D.E. (2021). Treatment of advanced melanoma in 2020 and beyond. J. Invest Dermatol. 141, 23-31. https://doi.org/10. 1016/j.jid.2020.03.943.

Lambert, A.W., Pattabiraman, D.R., and Weinberg, R.A. (2017). Emerging biological principles of metastasis. Cell 168, 670-691. https://doi.org/10.1016/j.cell.2016.11.037.

Orgaz, J.L., Pandya, P., Dalmeida, R., Karagiannis, P., Sanchez-Laorden, B., Viros, A., Albrengues, J., Nestle, F.O., Ridley, A.J., Gaggioli, C., et al. (2014). Diverse matrix metalloproteinase functions regulate cancer amoeboid migration. Nat. Commun. 5, 4255. https://doi.org/10.1038/ ncomms 5255 .

Pandya, P., Orgaz, J.L., and Sanz-Moreno, V. (2017). Modes of invasion during tumour dissemination. Mol. Oncol. 11, 5-27. https://doi. org/10.1002/1878-0261.12019.

Pinner, S., Jordan, P., Sharrock, K., Bazley, L., Collinson, L., Marais, R., Bonvin, E., Goding, C., and Sahai, E. (2009). Intravital imaging reveals transient changes in pigment production and Brn2 expression during metastatic melanoma dissemination. Cancer Res. 69, 7969-7977. https://doi.org/10.1158/0008-5472.CAN-090781

Quintanilla, M., Montero-Montero, L., Renart, J. and Martín-Villar, E. (2019). Podoplanin in inflammation and cancer. Int. J. Mol. Sci. 20, 707. https://doi.org/10.3390/ijms20030707.

Rodriguez-Hernandez, I., Maiques, $\mathrm{O}$., Kohlhammer, L., Cantelli, G., Perdrix-Rosell, A Monger, J., Fanshawe, B., Bridgeman, V.L., Karagiannis, S.N., Penin, R.M., et al. (2020) WNT11-FZD7-DAAM1 signalling supports tumour initiating abilities and melanoma amoeboid invasion. Nat Commun 11, 5315 https://doi.org/10.1038/s41467-020-18951-2.

Sanz-Moreno, V., Gadea, G., Ahn, J., Paterson, H. Marra, P., Pinner, S., Sahai, E., and Marshall, C.J. (2008). Rac activation and inactivation control plasticity of tumor cell movement. Cell 135 , 510-523. https://doi.org/10.1016/j.cell.2008.09 043.

Sanz-Moreno, V., Gaggioli, C., Yeo, M Albrengues, J., Wallberg, F., Viros, A., Hooper, S. Mitter, R., Féral, C.C., Cook, M., et al. (2011). ROCK and JAK1 signaling cooperate to control actomyosin contractility in tumor cells and stroma. Cancer Cell 20, 229-245. https://doi.org/ 10.1016/j.ccr.2011.06.018.

Schneider, C., Rasband, W., and Eliceiri, K. (2012). $\mathrm{NIH}$ Image to ImageJ: 25 years of image analysis. Nat Methods 9, 671-675. https://doi.org/10. 1038/nmeth.2089.

Shen, Y., Chen, C.S., Ichikawa, H., and Goldberg, G.S. (2010). Src induces podoplanin expression to promote cell migration. J. Biol. Chem. 285, 96499656. https://doi.org/10.1074/jbc.M109.047696.

Tozluoğlu, M., Tournier, A.L., Jenkins, R.P., Hooper, S., Bates, P.A., and Sahai, E. (2013). Matrix geometry determines optimal cancer cell migration strategy and modulates response to interventions. Nat. Cell Biol 15, 751-762. https:// doi.org/10.1038/ncb2775.

de Winde, C.M., Makris, S., Millward, L.J., Cantoral-Rebordinos, J.A., Benjamin, A.C. Martínez, V.G., and Acton, S.E. (2021). Fibroblastic reticular cell response to dendritic cells requires coordinated activity of podoplanin, CD44 and CD9. J. Cell Sci. 134, jcs258610. https:// doi.org/10.1242/jcs.258610.

Wyckoff, J.B., Pinner, S.E., Gschmeissner, S., Condeelis, J.S., and Sahai, E. (2006). ROCK- and myosin-dependent matrix deformation enables protease-independent tumor-cell invasion in vivo. Curr. Biol. 16, 1515-1523. https://doi.org/ 10.1016/j.cub.2006.05.065 


\section{STAR $\star$ METHODS}

\section{KEY RESOURCES TABLE}

\begin{tabular}{|c|c|c|}
\hline REAGENT or RESOURCE & SOURCE & IDENTIFIER \\
\hline \multicolumn{3}{|l|}{ Antibodies } \\
\hline anti-mouse podoplanin-biotin, clone 8.1.1 & eBioscience & $13-5381-82$ \\
\hline anti-mouse podoplanin & Acris antibodies & DM3501 \\
\hline anti-phospho-Myosin Light Chain 2 (Ser19) & Cell Signaling Technology & $3671 \mathrm{~L}$ \\
\hline anti-phospho-Ezrin/Radixin/Moesin & Cell Signaling Technology & $3141 \mathrm{~L}$ \\
\hline Phalloidin-AlexaFluor 647 & Sigma-Aldrich & P1951-1MG \\
\hline anti-mouse podoplanin-eFluor660 & eBioscience & $50-5381-82$ \\
\hline anti-Podoplanin-PE & BD Biosciences & 566390 \\
\hline Annexin V (APC/Fire $\left.{ }^{T M} 750\right)$ & Biolegend & 640953 \\
\hline 7-AAD & Biolegend & 420404 \\
\hline $\begin{array}{l}\text { anti-Phospho-Myosin Light Chain } 2 \text { (Thr18/ } \\
\text { Ser19) }\end{array}$ & Cell Signaling Technology & $3674 \mathrm{~L}$ \\
\hline anti-Myosin Light Chain 2 & Cell Signaling Technology & $3672 \mathrm{~L}$ \\
\hline anti-MelanA & Abcam & ab210546 \\
\hline anti-Tyrosinase & Santa Cruz & sc-20035 \\
\hline anti-Tubulin & Sigma-Aldrich & T7816 \\
\hline anti-GAPDH & Millipore & MAB374 \\
\hline anti-Histone $\mathrm{H} 3$ & Abcam & $a b 24824$ \\
\hline \multicolumn{3}{|l|}{ Chemicals, peptides, and recombinant proteins } \\
\hline ROCK inhibitor (GSK269962A) & MedChemExpress & HY-15556 \\
\hline \multicolumn{3}{|l|}{ Experimental models: Cell lines } \\
\hline WM983A & Prof. V sanz-Moreno & N/A \\
\hline WM983B & Prof. V sanz-Moreno & N/A \\
\hline 5555 & Prof. R Marais & N/A \\
\hline B16F10 & Francis Crick Institute & N/A \\
\hline \multicolumn{3}{|l|}{ Experimental models: Organisms/strains } \\
\hline C57BL/6J mice & Charles River Laboratories & Strain 027 \\
\hline \multicolumn{3}{|l|}{ Oligonucleotides } \\
\hline Details of qPCR primers are in Table S1 & This paper & N/A \\
\hline \multicolumn{3}{|l|}{ Recombinant DNA } \\
\hline GAP43-mCherry & Francis Crick Institute & \\
\hline pRP[CRISPR]-hCas9-U6>\{PDPN gRNA 1\} & Vector builder & VB160517-1061kpr \\
\hline $\begin{array}{l}\text { pPB[Exp]-mCherry/Bsd-TRE3G>mPdpn } \\
\text { [ORF036149]:P2A:CyPet }\end{array}$ & Vector builder & VB151008-10006 \\
\hline PDPN-CFP & Acton lab & N/A \\
\hline \multicolumn{3}{|l|}{ Software and algorithms } \\
\hline ImageJ & Schneider et al., 2012 & https://imagej.nih.gov/ij/ \\
\hline Imaris software & https://imaris.oxinst.com & \\
\hline QuPath 0.2.0 & https://doi.org/10.1038/s41598-017-17204-5 & https://qupath.github.io \\
\hline
\end{tabular}




\begin{tabular}{|c|c|c|}
\hline \multicolumn{3}{|l|}{ Continued } \\
\hline REAGENT or RESOURCE & SOURCE & IDENTIFIER \\
\hline \multicolumn{3}{|l|}{ Other } \\
\hline $\begin{array}{l}\text { Melanoma patient public studies - Kabbarah } \\
\text { et al. }\end{array}$ & Gene Expression Omnibus & GSE46517 \\
\hline Melanoma patient public studies - Riker et al. & Gene Expression Omnibus & GSE7553 \\
\hline Melanoma cell lines - Mannhein cohort & Gene Expression Omnibus & GSE4843 \\
\hline
\end{tabular}

\section{RESOURCE AVAILABILITY}

\section{Lead contact}

Further information and requests for resources and reagents should be directed to and will be fulfilled by the Lead Contact, Sophie E. Acton (s.acton@ucl.ac.uk).

\section{Materials availability}

Key resources including details of key reagents and cell lines used and generated are available in the Key Resources Table. Any unique reagents generated in this study are available from the Lead Contact with a completed Materials Transfer Agreement. Datasets are listed in the key resources table.

\section{Data and code availability}

- This paper analyzes existing, publicly available data. These accession numbers for the datasets are listed in the key resources table.

- This paper does not report original code

- Any additional information required to reanalyze the data reported in this paper is available from the lead contact upon request.

Links to publicly accessible datasets used in this study are listed in the key resources table. There is no code associated with this study.

\section{EXPERIMENTAL MODEL AND SUBJECT DETAILS \\ Mice}

Wild-type C57BL/6J mice were purchased from Charles River Laboratories. Both males and females were used for in vivo experiments and were aged 6-10 weeks. All mice were age-matched and housed in specific pathogen-free conditions. All animal experiments were reviewed and approved by the Animal and Ethical Review Board (AWERB) within University College London and approved by the UK Home Office in accordance with the Animals (Scientific Procedures) Act 1986 and the ARRIVE guidelines.

\section{METHOD DETAILS}

\section{Animal procedures}

Tumour growth. mOrange-labelled PDPN ${ }^{+}$and/or CFP-labelled PDPN KO B16F10 cells were injected subcutaneously into the flank of wild-type C57BL/6J mice (total $1 \times 10^{6}$ cells in $100 \mu \mathrm{l} \mathrm{PBS}$ per mouse). Tumours were excised 9-10 days after injection, and individual tumours were weighed. Tumours were fixed in AntigenFix (DiaPath, via Solmedia) overnight $\left(\mathrm{o} / \mathrm{n}\right.$ ) at $4^{\circ} \mathrm{C}$, and subsequently transferred to $30 \%$ sucrose for $\mathrm{o} / \mathrm{n}$ incubation at $4^{\circ} \mathrm{C}$. Tumours were frozen in O.C.T Compound (Tissue-Tek) using dry ice and isopentene, and stored at $-80^{\circ} \mathrm{C}$.

Intravital imaging. C57BL/6J mice were injected subcutaneously with 1:1 mix of PDPN ${ }^{10} 5555$-mCherry and PDPN-CFP transfected 5555-mCherry cells. When tumours were $3-7 \mathrm{~mm}$ diameter, mice were anaesthetised using isofluorane and a skin flap was cut to expose the tumour before the mouse was positioned on an Zeiss880 laser scanning microscope connected to a Chameleon Coherent Ti-Sapphire laser tuned to $850 \mathrm{~nm}$. Anaesthesia was maintained while time-lapse movies were made of the tumours. Each region was imaged for 20-30 min for each tumour. Following acquisition, timelapse stacks were drift corrected and 
movies are presented as z-projections using Imaris software. Analysis of cell movement and surface rendering was performed using spots and surfaces function in Imaris software.

Cell lines. WM983A (primary tumour) and WM983B (invasive/metastatic) human melanoma cell lines are previously described (Cantelli et al., 2015). Murine melanoma cell lines 4434 and 5555 (kind gift from Prof. Dr. R. Marais, The University of Manchester, Manchester, UK), and B16F10 (kind gift from Prof. Dr. C. Reis e Sousa, The Francis Crick Institute, London, UK) are all originally generated in C57BL/6 mice. B16F10 cell lines stably expressing mOrange or CFP were generated using the piggyBac transposon-based expression system. B16F10 cells were transfected with pBSR2-mOrange or pBSR2-CFP, and pBase plasmids (kind gift from Dr. E. Sahai, The Francis Crick Institute, London, UK). 5555 cell lines were labelled with mCherry membrane marker (GAP43-mCherry (kind gift from Dr. E. Sahai, The Francis Crick Institute, London, UK)) for intravital imaging. Additionally, PDPN-CFP (Acton et al., 2014) was overexpressed for gain-of-function experiments. To inhibit ROCK, cells were treated with $1 \mu \mathrm{M}$ GSK269962A or vehicle control (DMSO) for indicated time points.

Stable podoplanin knock-out (PDPN KO) B16F10 and podoplanin-inducible 5555 cell lines were generated using CRISPR/Cas9 editing. B16F10 cell lines were transfected with pRP[CRISPR]-hCas9-U6>\{PDPN gRNA 1\} plasmid (constructed and packaged by Vectorbuilder; vector ID: VB160517-1061 kpr) before negative selection using MACS LD columns (Miltenyi Biotec), anti-mouse podoplanin-biotin antibody $(50 \mu \mathrm{g} / \mathrm{mL}$, clone 8.1.1, eBioscience, 13-5381-82), and anti-biotin microbeads (Miltenyi Biotec, 130-090-485). To generate 5555 inducible PDPN/CyPet cells, 5555 cell lines were transfected with pPB[Exp]-mCherry/BsdTRE3G > mPdpn[ORF036149]:P2A:CyPet plasmid (constructed and packaged by Vectorbuilder; vector ID: VB151008-10006). To induce podoplanin and CyPET expression, 5555 inducible PDPN/CyPet cells were treated with $1 \mu \mathrm{g} / \mathrm{mL}$ doxycycline (DOX) for $48 \mathrm{~h}$ at $37^{\circ} \mathrm{C}, 10 \% \mathrm{CO}_{2}$.

Primary murine lymph node stromal cells were isolated and cultured for 3 days ex vivo (Acton et al., 2014). Murine and human melanoma cell lines were cultured in DMEM with GlutaMAX (Gibco, via Thermo Fisher Scientific) supplemented with 10\% fetal bovine serum (Sigma-Aldrich) and 100U/mL penicillin-streptomycin (Gibco, via Thermo Fisher Scientific) at $37^{\circ} \mathrm{C}, 5 \% \mathrm{CO}_{2}$ (B16F10) or $10 \% \mathrm{CO}_{2}$, and passaged using Trypsin/dPBS (Gibco, via Thermo Fisher Scientific).

\section{In vitro cell proliferation assay}

mOrange or CFP-labelled PDPN ${ }^{+}$or PDPN KO B16F10 cells $\left(5 \times 10^{4}\right.$ cells per well) were seeded in individual wells in 6-well plates (1 plate per time point). Every 24 hours, cells were harvested and diluted 1:2 in $0.4 \%$ Trypan Blue Solution (Thermo Fisher Scientific), and counted using a Neubauer Haemocytometer Counting Chamber (0.1 mm; Camlab).

\section{In vitro proteolysis assay}

12-well glass-bottomed culture plate (MatTek) was coated with Oregon Green 488-conjugated gelatin (Invitrogen; diluted 1:8 in solution of $2.5 \%(\mathrm{w} / \mathrm{w})$ gelatin/2.5\% $(\mathrm{w} / \mathrm{w})$ sucrose (Sigma-Aldrich)) for $10 \mathrm{~min}$ at room temperature (RT). B16F10 cells were seeded $\left(1.2 \times 10^{4}\right.$ cells per well) and incubated at $37^{\circ} \mathrm{C}, 5 \%$ $\mathrm{CO}_{2}$. After 20 hours, cell cultures were fixed with $3.6 \%$ formaldehyde (Sigma-Aldrich; diluted in PBS), and permeabilized with $0.2 \%$ Triton-X100 (Sigma-Aldrich) in PBS for 15min at RT. Cell nuclei were visualized using Hoechst (1:1000 dilution, Fisher Scientific) by $1 \mathrm{~h}$ incubation at RT. Stained samples were stored in PBS at $4^{\circ} \mathrm{C}$ until imaging.

\section{Immunofluorescence imaging \& analysis}

For analysis of cell morphology of WM983 cell lines, $2 \times 10^{4}$ cells were seeded on top of $1.7 \mathrm{mg} / \mathrm{mL}$ collagen (type I, rat tail) with or without Matrigel matrix (both from Corning, via Thermo Fisher Scientific) supplemented with $10 \%$ minimum essential medium alpha medium (MEMalpha, Invitrogen, via Thermo Fisher Sci-

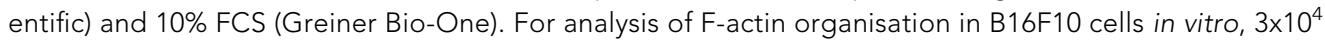
cells were seeded in $35 \mathrm{~mm}$ glass-bottomed culture dishes (MatTek) at $37^{\circ} \mathrm{C}, 10 \% \mathrm{CO}_{2}$. At $24 \mathrm{~h}$, cell cultures were fixed with AntigenFix (DiaPath, via Solmedia) for $15 \mathrm{~min}$ at RT or 3.6\% formaldehyde (Sigma-Aldrich; diluted in PBS), and permeabilized with $0.2 \%$ Triton-X100 (Sigma-Aldrich) in PBS for 15min at RT. F-actin and cell nuclei were visualized using respectively phalloidin-TRITC (P1951-1MG) and DAPI (D9542-1MG; both 1:500 dilution, both from Sigma-Aldrich). 
B16F10 tumours were cyrosectioned at 20 $\mu \mathrm{m}$. Frozen tumour sections were air dried for 10min at RT, encircled with a PAP pen for immunostaining (Sigma-Aldrich) and fixed with AntigenFix (DiaPath, via Solmedia) for $20 \mathrm{~min}$ at RT. Tissue sections were permeabilised and blocked with $0.3 \%$ Triton, $1 \%$ mouse serum and $2 \%$ BSA in $0.1 \mathrm{M}$ Tris-HCL buffer ( $\mathrm{pH} 7.4)$ for $1 \mathrm{~h}$, and stained with hamster anti-mouse podoplanin (1:500; clone 8.1.1, DM3501, Acris Antibodies) $\mathrm{o} / \mathrm{n}$ at $4^{\circ} \mathrm{C}$, then incubated with goat-anti-hamster IgG AlexaFluor 647 (1:500; Invitrogen, via Thermo Fisher Scientific) and phalloidin-TRITC (1:500; P1951-1MG) for $2 h$ at RT, and mounted in Mowiol (Sigma-Aldrich).

Untreated (control) 5555 cells or DOX-treated 5555 inducible WT PDPN/CyPet cells were seeded in $1.7 \mathrm{mg} / \mathrm{mL}$ collagen (type I, rat tail) with Matrigel matrix (both from Corning, via Thermo Fisher Scientific) supplemented with $10 \%$ minimum essential medium alpha medium (MEMalpha, Invitrogen, via Thermo Fisher Scientific), 10\% FCS (Greiner Bio-One) and DOX $(1 \mu \mathrm{g} / \mathrm{mL})$. After $24 \mathrm{~h}$, cell cultures were fixed with AntigenFix (DiaPath, via Solmedia) for $3 \mathrm{~h}$ at RT followed by blocking and permeabilization with $2 \%$ BSA (Sigma-Aldrich), 0.2\% Triton (Sigma-Aldrich) in PBS for $2 \mathrm{~h}$ at RT. Cells were stained with hamster anti-mouse podoplanin (1:500; clone 8.1.1, DM3501, Acris Antibodies), and rabbit anti-phospho-Myosin Light Chain 2 (Ser19) (1:50; 3671L, Cell Signalling) or rabbit anti-phospho-Ezrin/Radixin/Moesin (1:100; 3141L, Cell Signalling) $\mathrm{o} / \mathrm{n}$ at $4^{\circ} \mathrm{C}$, then incubated with goat-anti-hamster IgG AlexaFluor 488 (1:500; Invitrogen, via Thermo Fisher Scientific, A-21110) and goat-anti-rabbit IgG AlexaFluor 555 (1:500; Invitrogen, via Thermo Fisher Scientific, A-21429) for 3h at RT. F-actin and cell nuclei were visualized using Phalloidin-AlexaFluor 647 (P1951-1MG) and DAPI (D9542-1MG respectively; both 1:500 dilution, both from Sigma-Aldrich which were added along with the secondary antibodies).

All immunofluorescence images were acquired using a Leica DMI6000 SP5 confocal microscope using HC PL FLUOTAR /0.3 10x air (WM983 cell lines), or HCX PL APO /1.25 40x or /1.4 63x oil (B16F10 and 5555 cell lines and tumour sections) objective lenses.

Immunofluorescence images were analysed using Fiji/ImageJ software. Z stacks were projected using Z Project (maximum projection). Roundness index, cell area and perimeter were analysed by manually drawing around the cell shape using F-actin staining, or mOrange or CFP-labelling. Podoplanin or F-actin fluorescence intensity (mean gray value) in membrane blebs from 5555 PDPN-CFP-transfected cells was analysed using line plot function (average of three lines per bleb) measured from the cell body's cortex until outside the cell for the lifetime of the bleb.

Tilescans of tumour sections taken at various depths of mixed $\mathrm{PDPN}^{+} / \mathrm{KO}$ tumours from 2 mice were analysed using QuPath 0.2.0 (Figure S5). Using the pixel classifier tool, the software was trained based on defined PDPN ${ }^{+}$(mOrange), PDPN KO (CFP) and tumour-free regions. A pixel classification was then created and applied to all images, dividing sections into PDPN ${ }^{+}$, PDPN KO and tumour-free areas. Annotations were created around the whole tumour section, and overall proportions of PDPN ${ }^{+}:$PDPN KO were automatically generated. Invasive front sections were defined as $30 \mu \mathrm{m}$ from the tumour edge. The margin was classified where the bulk of tumour fluorescence ended.

\section{Flow cytometry}

Single-cell suspensions of murine melanoma cell lines were incubated with FcR blocking reagent (Miltenyi Biotec) as per supplier's instructions, followed by staining with hamster anti-mouse podoplanin-eFluor660 antibody (1:200, clone 8.1.1, eBioscience, 50-5381-82) diluted in PBS supplemented with 0.5\% BSA and 5mM EDTA for $30 \mathrm{~min}$ on ice. Stained cells were analysed using FACSDiva software and LSRII flow cytometer (BD Biosciences). Flow cytometry data was analysed using FlowJo Software version 10 (BD Biosciences).

\section{Apoptosis assay}

5555 melanoma cells were analysed following doxycycline induction of podoplanin. Cells were incubated with purified rat anti-mouse CD16/CD32 (Mouse BD Fc Block ${ }^{\mathrm{TM}}, 0.5 \mu \mathrm{L} /$ test, BD Biosciences, 553142) as per supplier's instructions. Cells were stained with hamster anti-Podoplanin-PE (clone 8.1.1 (RUO), 0.5 $\mu \mathrm{L} /$ test, BD Biosciences, 566390) diluted in PBS supplemented with 5\% BSA and 5mM EDTA for 30 min in dark at $4^{\circ} \mathrm{C}$. Cells were then stained using the Annexin V (APC/Fire ${ }^{\mathrm{TM}} 750$, Biolegend, 640953) and 7-AAD (Biolegend, 420404) as per supplier's instructions. Stained cells were analysed using the Fortessa X-20 flow cytometer (BD Biosciences). All flow cytometry data was analysed using FlowJo Software version 10 (BD Biosciences). 
RNA isolation and quantitative RT-PCR analysis

mRNA of primary lymph node stromal cells, WM983, and B16F10 cell lines was isolated using RNeasy Mini kit (Qiagen) as per supplier's instructions, including a DNA digestion step. Then reverse transcribed to cDNA using SuperScript ${ }^{\circledR}$ IV First-Strand Synthesis System kit (Invitrogen, via Thermo Fisher Scientific) as per supplier's instructions. Transcript abundance of genes of interest were determined with a CFX96 Sequence Detection System (Bio-Rad) with MESA Blue (Eurogentec, via Promega). Gene-specific oligonucleotide primers are listed in Table S1. Ct values of the genes of interest were normalized to the Ct value of the housekeeping gene GAPDH (WM983 cell lines), Pdgfra (lymph node stromal cells) or Gapdh (B16F10 cell lines). All primers are listed in Table S1.

\section{Western blotting}

Ctrl or CLEC-2-Fc FRCs were plated in a 6-well culture plate $\left(1 \times 10^{5}\right.$ cells per well). After 24 hours, the culture plate was placed on ice and cells were washed twice with cold PBS. Cells were lysed in $100 \mu \mathrm{L} 4 \times \mathrm{Laemmli}$ lysis buffer (Bio-Rad) and collected using a cell scraper. Samples were separated by reducing 10\% SDSpolyacrylamide gel electrophoresis. Western blots were incubated with rabbit anti-Phospho-Myosin Light Chain 2 (Thr18/Ser19) (ppMLC; 1:750, Cell Signaling Technology, 3674), rabbit anti-Myosin Light Chain 2 (MLC; 1:750, Cell Signaling Technology, 3672), rabbit anti-MelanA (EPR20380, 1:2000, Abcam, ab210546), mouse anti-human Tyrosinase (T311, 1:100, Santa Cruz, sc-20035), rabbit anti-Brn2/POU3F2 (D2CIL, 1:1000, Cell Signaling Technology, 12137), rabbit anti-podoplanin (1:1000, iSpyBio, ABT34), or as loading controls mouse anti-Tubulin (1:20,000, Sigma Aldrich, T7816), mouse anti-GAPDH (1:10,000, clone 6C5, Millipore, MAB374) or mouse anti-Histone H3 (1:2500, Abcam, ab24824), in PBS supplemented with $1 \%$ skim milk powder and $0.2 \%$ BSA overnight at $4{ }^{\circ} \mathrm{C}$, followed by staining with appropriate HRP-conjugated secondary antibodies (Abcam) for 2 hours at RT. Western blots were developed using Luminata Crescendo Western HRP substrate (Merck Millipore) and imaged on ImageQuant LAS 4000 mini (GE Healthcare Life Sciences).

\section{Melanoma patient and cell lines mRNA database analysis}

Expression data from melanoma patient public studies Kabbarah et al (GSE46517) and Riker et al (GSE7553) were extracted from GEO (Gene Expression Omnibus) database and normalized using Gene Pattern software (http://www.broadinstitute.org/cancer/software/genepattern/). Expression data from melanoma cell lines (Mannheim cohort; GSE4843) was obtained from the study Hoek et al., 2006. One outlier was excluded from the Mannheim cohort. Expression data from the TCGA database are based upon data generated by the TCGA Research Network: https://www.cancer.gov/tcga.

\section{QUANTIFICATION AND STATISTICAL ANALYSIS}

Statistical differences between two groups were determined using unpaired Student's t-test (two-tailed), or, in the case of non-Gaussian distribution, Mann-Whitney test. Statistical differences between more than two groups were determined using, in the case of non-Gaussian distribution, Kruskal-Wallis test with Dunn's multiple comparisons. Statistical differences between PDPN + cells in the whole tumour compared to the invasive front were determined using multiple $t$ tests. Podoplanin/pMLC or $p E R M$ expression was analysed using linear regression analysis. Statistical differences between expression of melanocyte and invasion-associated genes in PDPN ${ }^{+}$and PDPN KO B16F10 cells were determined using two-way ANOVA with Sidak's multiple comparisons test. Statistical tests were performed using GraphPad Prism software (version 7), and differences were considered to be statistically significant at (adjusted) $p \leq 0.05$. 